\title{
Chiral bifunctional sulfide-catalyzed asymmetric bromoaminocyclizations
}

Received 00th January 20xx, Accepted 00th January 20xx DOI: $10.1039 / x 0 x x 00000 x$

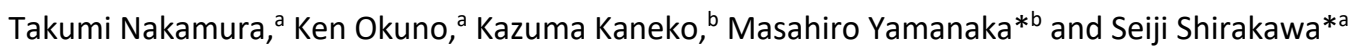

A BINOL-derived chiral bifunctional sulfide catalyst bearing a phenylurea moiety was applied to enantioselective bromoaminocyclization reactions of 2-allylaniline derivatives, which provide optically active 2-substituted indoline products as important motifs for biologically active compounds. A protecting group on the nitrogen of the 2-allylaniline substrate was carefully optimized, and highly enantioselective reactions were achieved by employing the $p$-biphenylsulfonyl-protected substrates. The origin of the good level of enantioselectivity for the present bromoaminocyclization was also investigated on the basis of DFT calculations. The resultant optically active 2-(bromomethyl)indoline products could be transformed to various 2-substituted indolines with no loss of the optical purity.

\section{Introduction}

Catalytic asymmetric bromolactonizations are some of the most effective methods used to construct important chiral lactones in optically active form. ${ }^{1}$ A wide variety of chiral metal- and organo-catalytic methods for stereoselective bromolactonizations have been reported. Despite the existence of several effective catalytic systems for stereoselective bromolactonizations and related halofunctionalizations, ${ }^{2}$ further developments of new chiral catalysts, which can be applied to various bromocyclizations, are still highly desired due to the importance of the resultant chiral lactones and related products. In this context, we have recently achieved highly regio-, diastereo-, and enantioselective bromolactonizations of stilbene-type carboxylic acids by utilizing a newly designed type (S)-1 chiral bifunctional sulfide catalyst (Scheme 1). $\cdot^{3-5}$ To demonstrate the further potential utility of chiral bifunctional sulfides (S)-1, we next became interested in the application to asymmetric bromoaminocyclizations of 2-allylanilines $\mathbf{2}$, which provide optically active 2-(bromomethyl)indolines 3.6,7 Herein, we report chiral bifunctional sulfide-catalyzed enantioselective bromoaminocyclizations for use in the synthesis of chiral indolines as an important structural motif in biologically active compounds. ${ }^{8}$
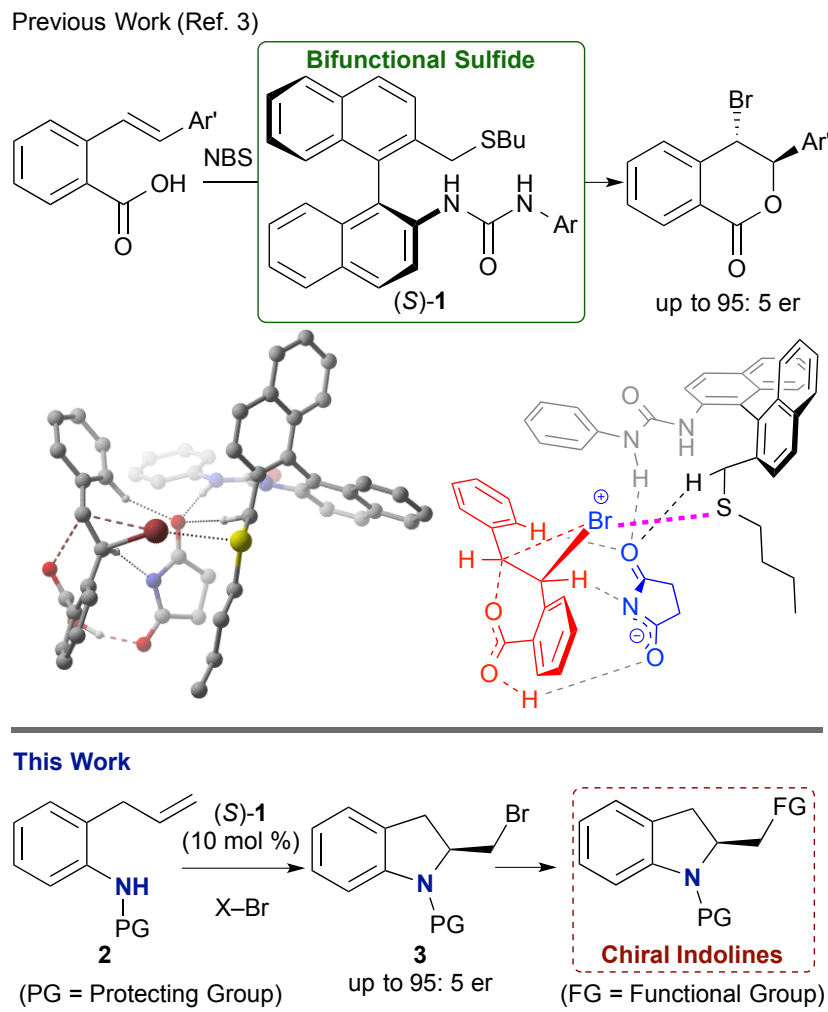

Scheme 1 Chiral bifunctional sulfides for asymmetric bromocyclizations.

\section{Results and discussion}

Environmental Sciences, Nagasaki University 1-14 Bunkyo-machi, Nagasaki 8528521, Japan.E-mail: seijishirakawa@nagasaki-u.ac.jp

b. Address here. Department of Chemistry and Research Center for Smart Molecules, Faculty of Science, Rikkyo University, 3-34-1, Nishi-lkebukuro, Toshima-ku, Tokyo 171-8501, Japan. E-mail: myamanak@rikkyo.ac.jp

† Footnotes relating to the title and/or authors should appear here.

Electronic Supplementary Information (ESI) available: [details of any supplementary information available should be included here]. See DOI: 10.1039/x0xx00000x
$\mathrm{N}$-Tosyl-2-allylaniline $2 \mathrm{a}$ was selected as a model substrate to evaluate the catalytic utility of chiral bifunctional sulfides (S)-1 for use in bromoaminocyclizations (Table 1). An attempted reaction of $\mathbf{2 a}$ with $N$-bromophthalimide (NBP) in 
dichloromethane under the influence of a phenylurea-type bifunctional sulfide $(S)-1$ a at $-78^{\circ} \mathrm{C}$ for $24 \mathrm{~h}$ provided $\mathrm{N}$-tosyl-2(bromomethyl)indoline $\mathbf{3 a}$ in a good yield with moderate enantioselectivity (78:22 er; entry 1$)$. Tunings the urea moiety on catalyst (S)-1 did not improve the results (entries 2 and 3). Other BINOL-derived sulfide catalysts (S)-4 were also examined. ${ }^{3 c}$ The reaction with bifunctional sulfide (S)-4a bearing a hydroxy group provided product $\mathbf{3 a}$ in poor enantioselectivity with a configuration that was the opposite of a major isomer (46:54 er; entry 4). Methoxy-substituted chiral sulfide $(S)-\mathbf{4 b}$ also gave product $\mathbf{3 a}$ with a very low level of enantioselectivity (51:49; entry 5). As a result of the screening of our BINOL-derived sulfide catalysts, we selected the chiral bifunctional sulfide (S)-1a, which possesses a phenylurea moiety, as a promising catalyst for further optimization of the bromoaminocyclization. It should be noted that the present reaction proceeded even without a catalyst (entry 6).

Table 1 Catalyst optimization ${ }^{a}$

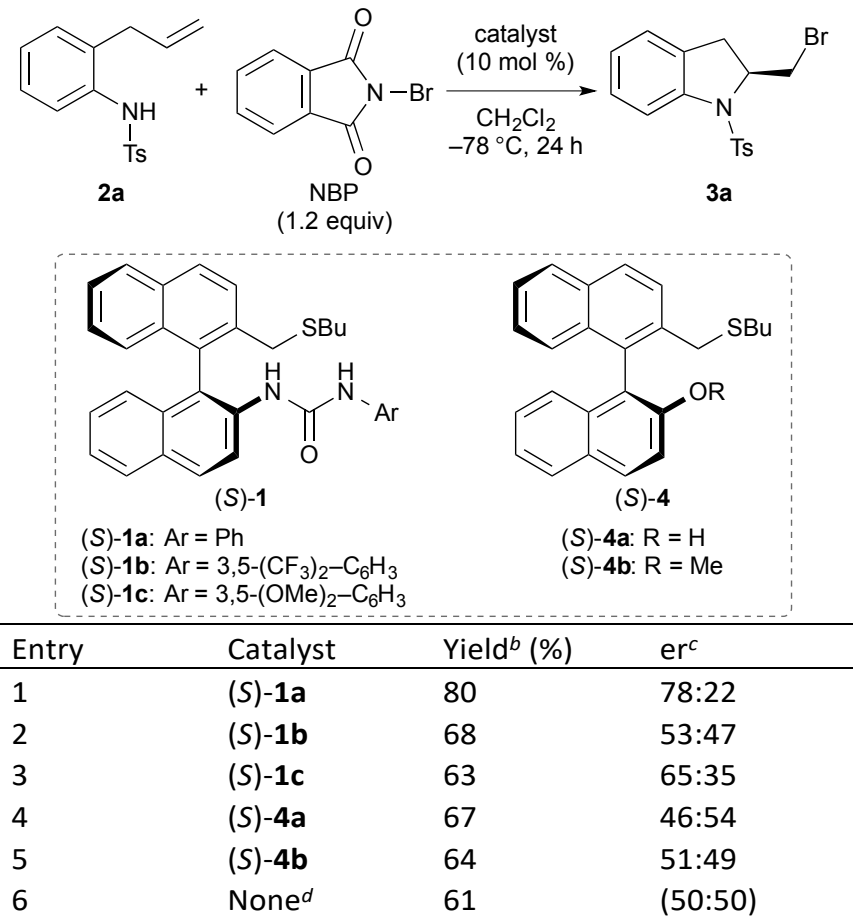

a Reaction conditions: 2a $(0.10 \mathrm{mmol}), \mathrm{NBP}(0.12 \mathrm{mmol})$, catalyst $(10 \mathrm{~mol} \%, 0.010 \mathrm{mmol}), \mathrm{CH}_{2} \mathrm{Cl}_{2}(2.0 \mathrm{~mL}),-78^{\circ} \mathrm{C}, 24 \mathrm{~h} .{ }^{b}$ Yield of isolated product 3a. ${ }^{c}$ Enantiomeric ratio (er) was determined by HPLC analysis on a chiral stationary phase. ${ }^{d}$ The reaction was performed without a catalyst.

Next, we examined the effects of brominating reagents (Table 2). Reactions using a catalyst (S)-1a with brominating reagents possessing 5-membered ring structures, except $\mathrm{N}$ bromosaccharin (NBSac), gave product $\mathbf{2} \mathbf{a}$ in constant levels of enantioselectivity (entries 1-3). The reaction with NBSac as a higher reactive brominating reagent showed lower enantioselectivity probably due to the promotion of an undesired background reaction (entry 4). Other brominating reagents with 6-membered rings and acyclic structures provided product 3a in levels of enantioselectivity that were lower than that of the reaction using NBP (entries 5-7). The reaction with bromine $\left(\mathrm{Br}_{2}\right)$ produced $3 a$ as a racemate (entry 8). Among the examined brominating reagents, NBP showed the best enantioselectivity (entry 2 ).

Table 2 Effect of brominating reagents ${ }^{a}$

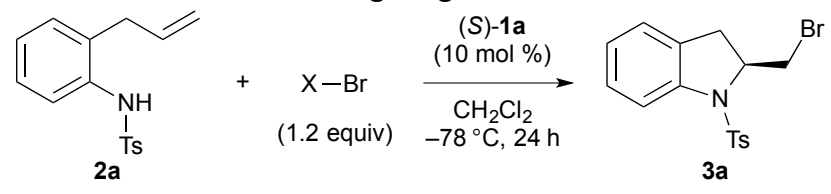

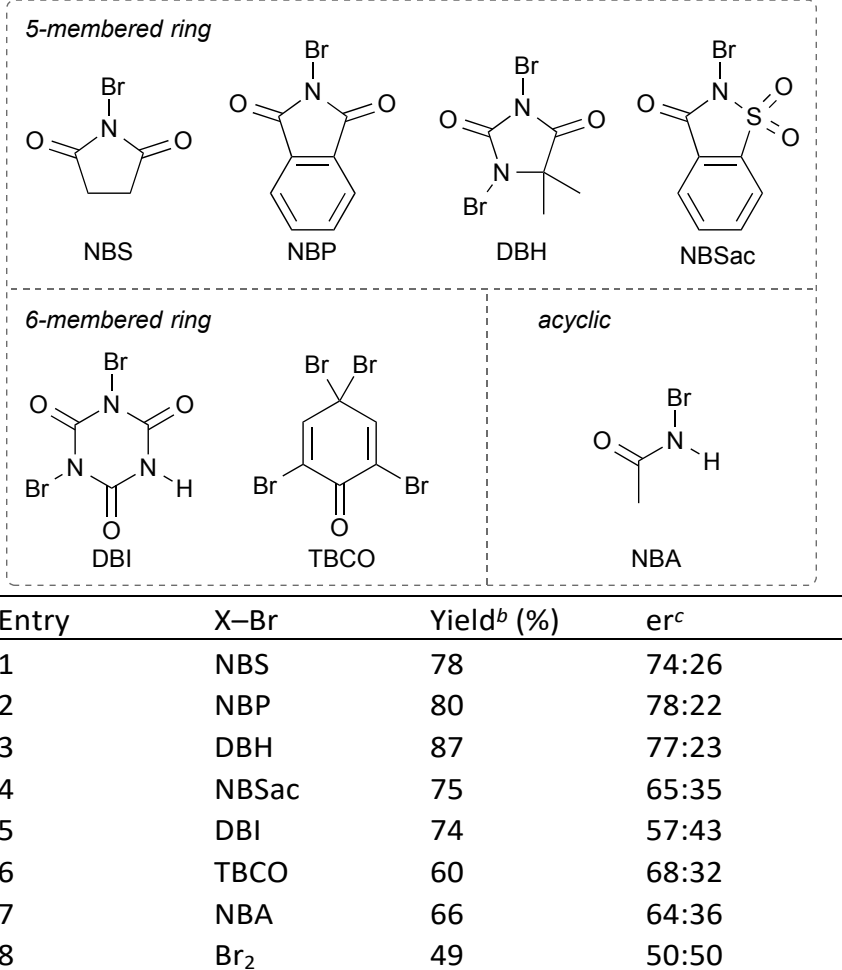

a Reaction conditions: $2 \mathrm{a}(0.10 \mathrm{mmol})$, brominating reagent (0.12 mmol), (S)-1a (10 mol \%, $0.010 \mathrm{mmol}), \mathrm{CH}_{2} \mathrm{Cl}_{2}(2.0 \mathrm{~mL}),-$ $78{ }^{\circ} \mathrm{C}, 24 \mathrm{~h} .{ }^{b}$ Yield of isolated product 3a. ${ }^{c}$ Enantiomeric ratio (er) was determined by HPLC analysis on a chiral stationary phase.

To improve the enantioselectivity of the present bromoaminocyclization of 2-allylanilines $\mathbf{2}$, next we carefully investigated the structural effects of the $N$-protecting group on substrate 2 (Scheme 2). First, we examined the effect on the position of the methyl group in an $N$-toluenesulfonyl moiety. By comparison with $p$-toluenesulfonyl (Ts)-protected substrate $\mathbf{2 a}$ (78:22 er for 3a), the reactions with $m$ - and $o$-toluenesulfonylsubstituted substrates $\mathbf{2} \mathbf{b}$ and $\mathbf{2 c}$ provided products $\mathbf{3 b}$ and $\mathbf{3 c}$ in lower enantioselectivities. Additionally, benzenesulfonylprotected substrate $\mathbf{2 d}$ showed enantioselectivity that was slightly lower (70:30 er for $\mathbf{3 d}$ ) than that of $\mathbf{2 a}$. To check the effect of the electronic nature on the $N$-protecting groups, we also examined $p$-nitrobenzenesulfonyl-substituted substrate $\mathbf{2 e}$. Interestingly, the reaction with $\mathbf{2 e}$ provided product $\mathbf{3 e}$ as a racemate. Based on these tendencies, we then turned to an 
examination of the steric effect on arylsulfonyl at the paraposition. Fortunately, the reaction with p-tertbutylbenzenesulfonyl-substituted substrate $\mathbf{2 f}$ improved the enantioselectivity (81:19 er for $\mathbf{3 f}$ ). Encouraged by this result, we also prepared the $p$-biphenylsulfonyl-protected substrate $\mathbf{2 g}$. To our delight, the reaction of $\mathbf{2} \mathbf{g}$ provided a satisfactory result and we could obtain 2-(bromomethyl)indoline $\mathbf{3 g}$ in good enantioselectivity (93: $7 \mathrm{er}$ ). The reaction of $\mathbf{2 g}$ at lower temperature $\left(-90^{\circ} \mathrm{C}\right)$ further improved the enantioselectivity while maintaining a high yield (89\% yield, 95 : 5 er for $\mathbf{3 g}$ ).
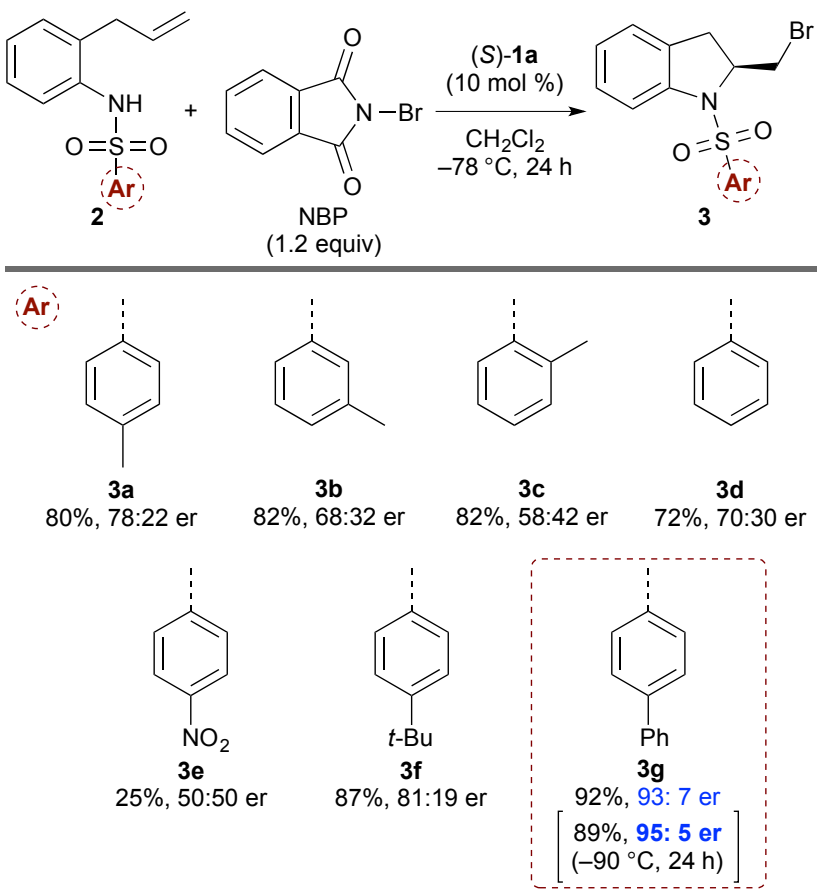

Scheme 2 Fine optimization of protecting groups.

With the optimum catalyst (Table 1 ), brominating reagent (Table 2), and protecting group on the substrate (Scheme 2 ) in hand, we conducted DFT calculations $\left[\mathrm{SMD}\left(\mathrm{CH}_{2} \mathrm{Cl}_{2}\right)-\omega \mathrm{B} 97-\right.$ XD/def2TZVP//SMD $\left.\left(\mathrm{CH}_{2} \mathrm{Cl}_{2}\right)-\omega B 97 X D / 6-31 \mathrm{G}^{*}\right]^{9}$ of the present bromoaminocyclization of $\mathbf{2 g}$ to computationally investigate the origin of the good enantioselectivity. Based on our previous transition state (TS) model, ${ }^{3 a}$ we explored various TS models consisting of (S)-1a, NBS, and $\mathbf{2 g}$ (Fig. S1 in ESI). ${ }^{10}$ After the preliminary screening of TS models, it was found that the major and minor enantiomers were obtained through concerted and stepwise pathways, respectively (Fig. 1). For the concerted pathway via TS1s leading to the major enantiomer, (S)-1a synergistically activates NBS and $\mathbf{2} \mathbf{g}$ to simultaneously form the $\mathrm{C}-\mathrm{N}$ and $\mathrm{C}-\mathrm{Br}$ bonds. By contrast, in the stepwise pathway via TS2 $r$ and TS3r leading to the minor enantiomer, bromination proceeds in the first step (TS2r) and cyclization occurs via the resultant bromonium intermediate (INTr) in the second step (TS3r). The energetically less stable TS3r is the rate- and stereoselectivity-determining step in the stepwise pathway. TS1s is $1.4-\mathrm{kcal} / \mathrm{mol}$ more stable than TS3r in a qualitatively good agreement with the experimental results. Both TS1s and
TS3r have similar structural features regarding the orientation of $\mathbf{2 g}$ and NBS toward (S)-1a, hydrogen bonds, and other attractive non-covalent interactions (Fig. 2).

The only difference in the cyclic TS is the facial selection of the olefinic moiety of $\mathbf{2 g}$. To clarify the major factor in the energy difference between TS1s and TS3r, partially defected TS models (TS-d1 and -d2: the sulfide group and the Ph group of the urea moiety are exchanged with $\mathrm{H}$ atom, TS-d3: the succinimide anion is removed) were investigated (Fig. S2 in ESI). ${ }^{10}$ TS1s-d1 is found to be less stable than TS3r-d1 in contrast to the relatively small change of the energy difference in TS-d2 and $-\mathbf{d} \mathbf{3}$ models. The $\mathrm{S}-\mathrm{Br}$ halogen bonding interaction in the bromosulfonium moiety of (S)-1a plays a key role in stabilizing TS1s compared with the succinimide anion and the terminal $\mathrm{Ph}$ group of the urea moiety in (S)-1a which induce the $\pi / \pi$, $\mathrm{CH} / \pi$, and hydrogen bonding interactions. Such computational results well consist with the importance of the sulfide-urea bifunctionality of (S)-1a (Table 1). The great impact on the $\mathrm{S}-\mathrm{Br}$ halogen bonding interaction is clearly caused by the concerted TS structure of TS1s in which the S-Br bond is partially broken, whereas the $\mathrm{S}-\mathrm{Br}$ bond is completely broken in TS3r. The hydrogen-bonding complexes (CPs and CPr) leading to TS1s and TS2r allow us to demonstrate the role of the $N$-protecting group in differentiating the reaction pathways (Fig. 3).

In both CPs and CPr, the $p$-biphenylsulfonyl group arranges perpendicular to the $\mathrm{Ph}$ group of the urea moiety to fix the orientation of $2 \mathrm{~g}$ by $\mathrm{CH} / \pi$ and $\pi / \pi$ interactions among the aryl groups. Furthermore, the $\mathrm{NH}-\mathrm{O}$ and $\mathrm{CH}-\mathrm{N}$ hydrogen bonds between $\mathbf{2 g}$ and the succinimide anion control the rotational position of the $\mathrm{N}$-sulfonylamine group of $\mathbf{2 g}$ (highlighted in green, Fig. 3). The $N$-sulfonylamine group is positioned more perpendicular to the benzene ring to enhance the nucleophilicity of the amine group in CPs, while the lone pair electrons of the amino group are more delocalized in CPr. To undergo cyclization ( $\mathrm{C}-\mathrm{N}$ bond formation), more conjugated and less nucleophilic amino group in CPr would require a highly activated olefin such as a bromonium intermediate. Therefore, the attractive non-covalent interaction network between $\mathbf{2 g}$ and $(S)-\mathbf{1 a} /$ NBS controls the orientation of $\mathbf{2 g}$ toward $(S)-\mathbf{1 a}$, enhancing the nucleophilicity of the amino group and promoting the energetically favored concerted pathway via TS1s. These computational results well explained the origin of the good enantioselectivity as well as the roles of the sulfideurea bifunctionality of (S)-1a and the $N$-protecting group of $\mathbf{2}$. 


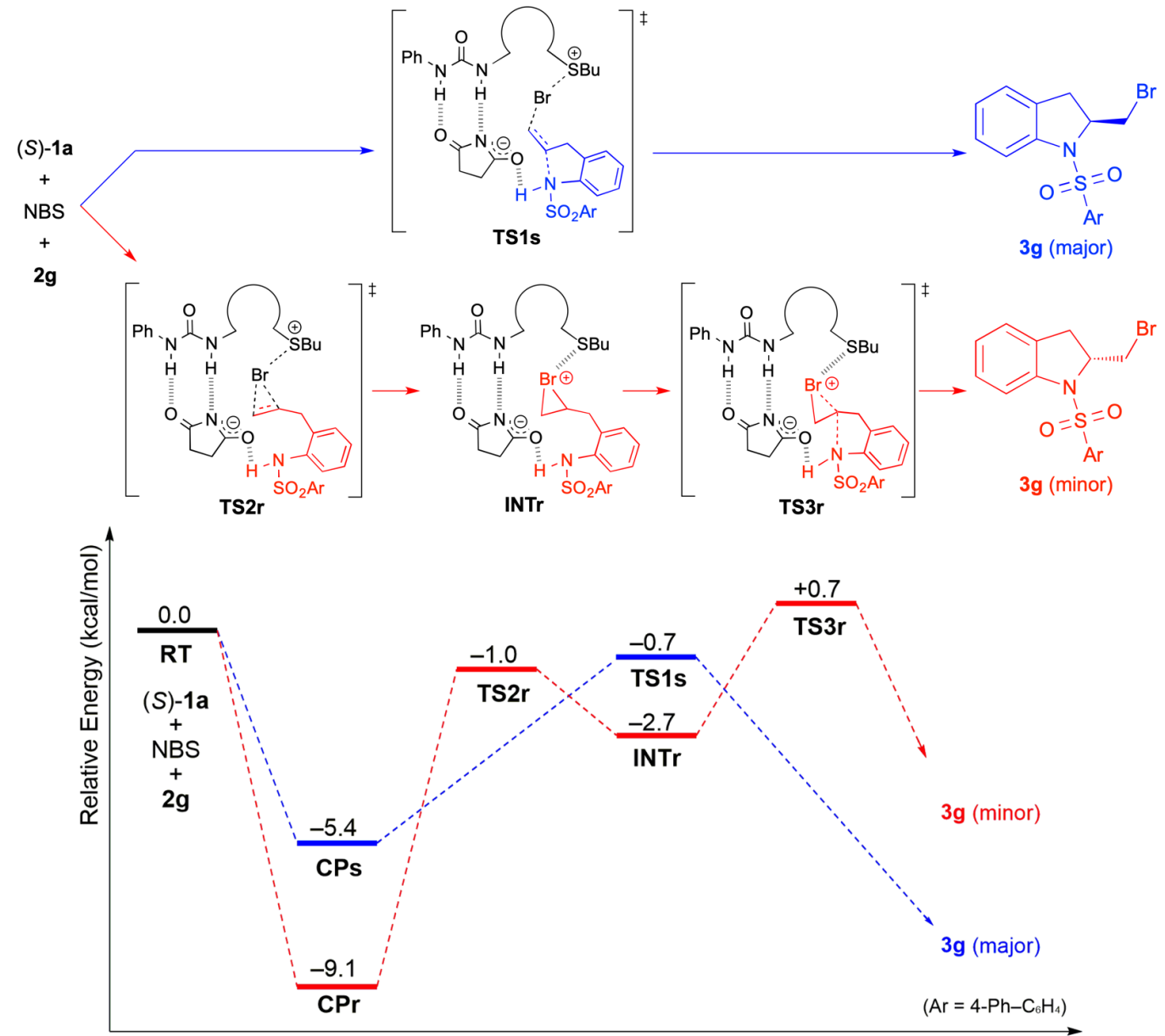

Fig. 1 Energy profile for the bromoaminocyclization of $\mathbf{2 g}$. The sum of the energies of (S)-1a, NBS, and $\mathbf{2} \mathbf{g}$ are set to zero. Bond lengths are in $\AA$.
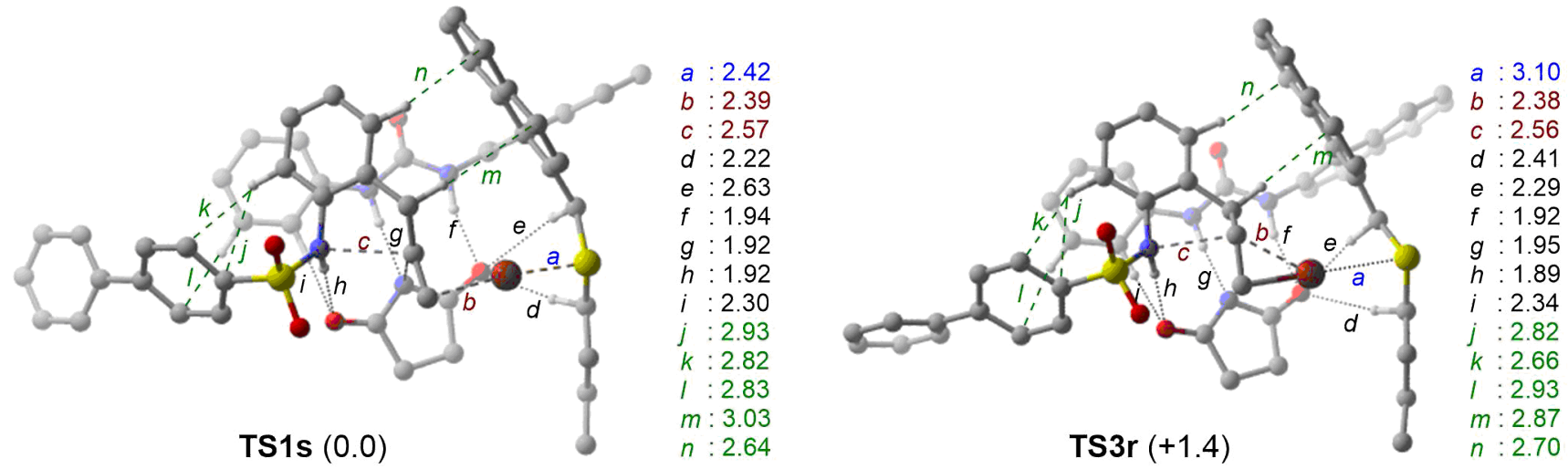

Fig. 2 The 3D structures and the relative energies $(\mathrm{kcal} / \mathrm{mol})$ of TS1s and TS3r. Distances are shown in Å. 


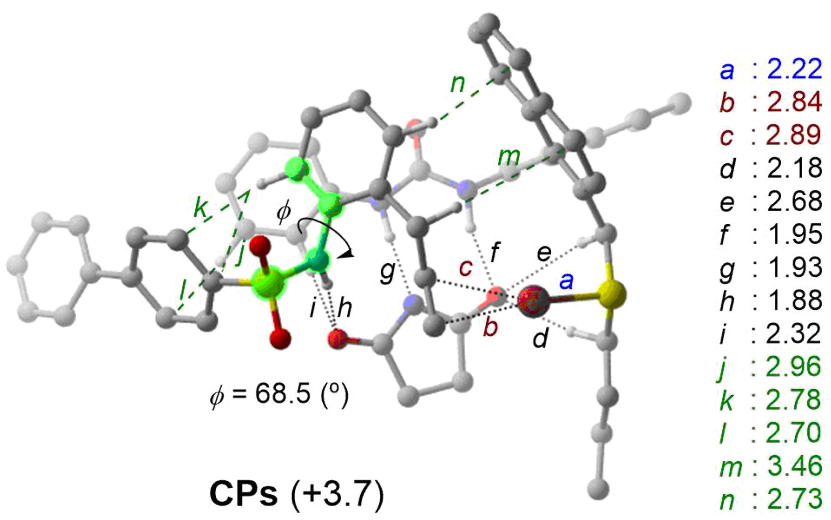

Fig. 3 The 3D structures and the relative energies $(\mathrm{kcal} / \mathrm{mol})$ of CPs and CPr. Distances are shown in $\AA$.

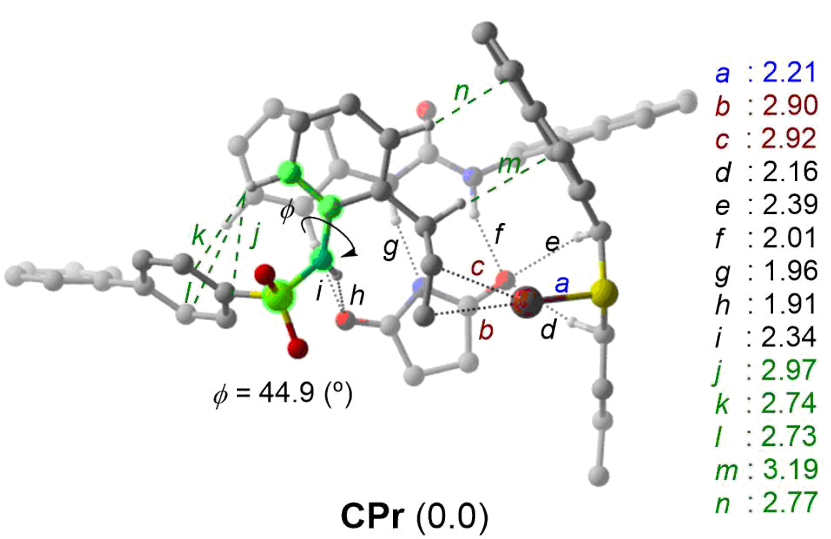

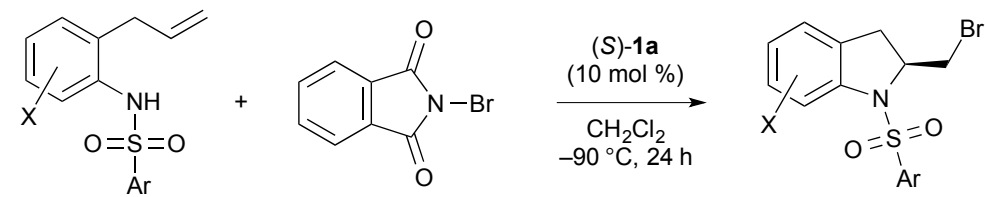

${ }_{5}^{\mathrm{Ar}}$

$\left(\mathrm{Ar}=\stackrel{\mathbf{5}}{\mathrm{Ph}}-\mathrm{C}_{6} \mathrm{H}_{4}\right)$

NBP

(1.2 equiv)<smiles>Clc1ccc2c(c1)CC(CBr)N2</smiles>

$0=\stackrel{1}{S}=0$

6a

$77 \%, 88: 12$ er

$89 \%, 95: 5$ er

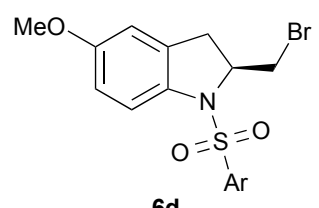

$82 \%, 89: 11$ er

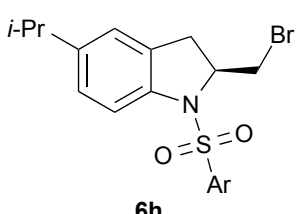

$98 \%, 87: 13$ er

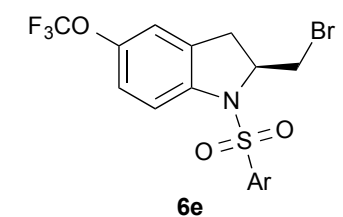

$49 \%, 84: 16$ er

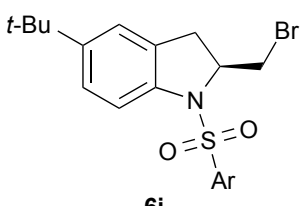

$6 i$
95\%, 93: 7 er

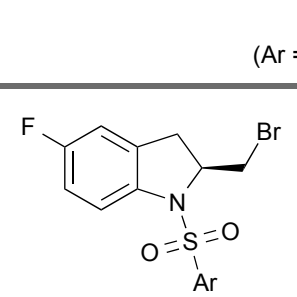

6b

85\%, 94 : 6 er

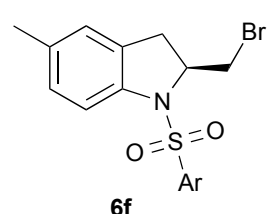

92\%, 93: 7 er

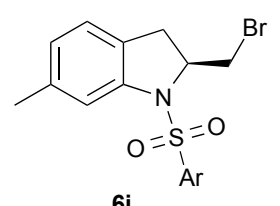

71\%, $73: 27$ er
6<smiles>O=S([Ga])(=[Se])N1c2ccc(C(F)(F)F)cc2C[C@H]1CBr</smiles>

$27 \%, 77: 23$ er

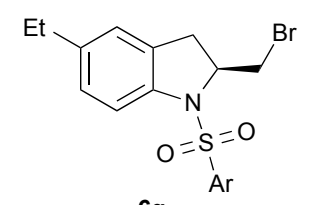

$6 \mathrm{~g}$

98\%, 92: 8 er

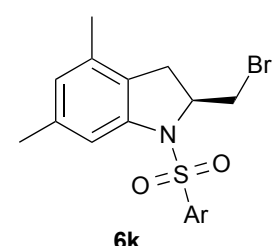

$90 \%, 75: 25$ er

Scheme 3 Substrate scope. 
We further studied the substrate scope of the bromoaminocyclization of $\mathrm{N}$-( $p$-biphenylsulfonyl)-2-allylaniline derivatives $\mathbf{5}$ (Scheme 3 ). When $\mathbf{5}$-halo-substituted allylanilines $\mathbf{5 a}$ and $\mathbf{5 b}$ were mixed with NBP under the influence of chiral bifunctional sulfide catalyst (S)-1a, the corresponding indoline products $\mathbf{6 a}$ and $\mathbf{6 b}$ were obtained in good yields and enantioselectivities. On the other hand, the reaction with 5trifluoromethyl-substituted allylaniline $\mathbf{5 c}$ provided product $\mathbf{6 c}$ in a low yield with only moderate enantioselectivity. ${ }^{11}$ The 5methoxy and trifluoromethoxy-substituted allylanilines $\mathbf{5} \mathbf{d}$ and $\mathbf{5 e}$, however, could be transformed to indolines $\mathbf{6} \mathbf{d}$ and $\mathbf{6 e}$ with good levels of enantioselectivity. The reactions with 5-alkylsubstituted substrates $\mathbf{5} \mathbf{f}-\mathbf{i}$ uniformly produced products $\mathbf{6} \mathbf{f}-\mathbf{i}$ in high yields with good enantioselectivities. Methyl-substituents at other positions caused a decrease in the enantioselectivity, and products $\mathbf{6} \mathbf{j}$ and $\mathbf{6 k}$ were obtained with only moderate levels of enantioselectivity.

To expand the substrate scope of catalytic asymmetric bromoaminocyclizations, related substrates were applied to our catalytic system (Scheme 4). The reaction with 2methallylaniline derivative $\mathbf{7}$ provided 2,2-disubstituted indoline product $\mathbf{8}$ in a good yield with moderate enantioselectivity. Also, 2-allylbenzylamine derivative $\mathbf{9}$ could be applied to the present reaction system to produce tetrahydroisoquinoline derivative $\mathbf{1 0}$ in a high yield with moderate enantioselectivity. Although the observed levels of enantioselectivity for these substrates were moderate, this catalytic system suggests wide applicability to the construction of other important heterocyclic amines. The levels of enantioselectivity may be able to improve with further optimization of the catalyst structure.
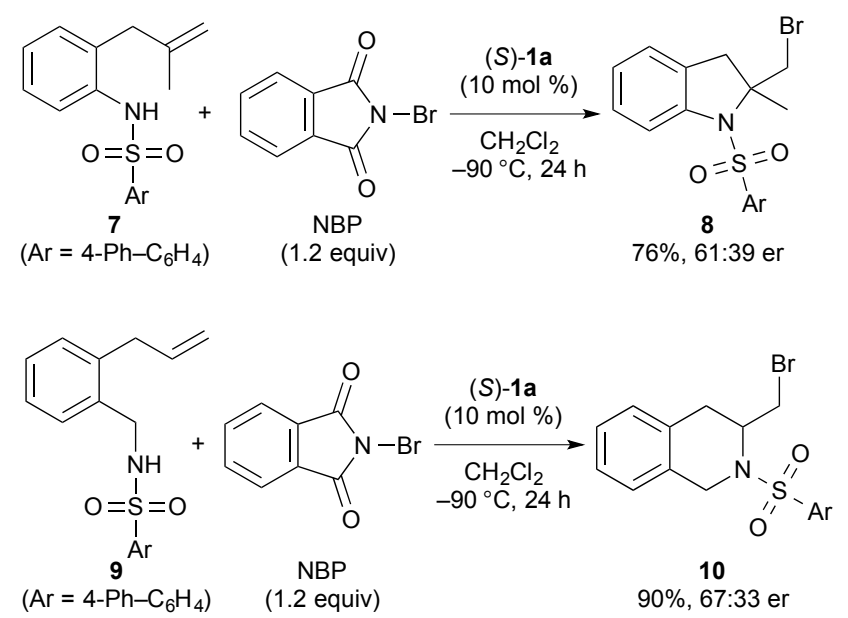

Scheme 4 Other bromoaminocyclizations.

The transformations of the 2-(bromomethyl)indoline $\mathbf{3 g}$ were examined to demonstrate the additional utility of the present asymmetric synthesis. Catalytic asymmetric bromoaminocyclization with $\mathbf{2} \mathbf{g}$ was performed on a larger scale (1.0 $\mathrm{mmol}$ scale) to obtain product $\mathbf{3 g}$ for further

transformations (Scheme 5). Basically, the same yield and enantioselectivity were observed when compared with a smaller scale reaction (see; Scheme 2). The bromo group on $\mathbf{~} \mathbf{g}$ was reduced under radical conditions using tributyltin hydride to provide $\mathbf{1 1}$. Optically active 2-methylindoline $\mathbf{1 2}$, which is a key building block for the preparation of several medicines, ${ }^{12}$ was synthesized by deprotection of the arylsulfonyl protecting group in 11. The introductions of nitrogen, sulfur, and oxygen functional groups were performed via nucleophilic substitutions, and products 13, 14, and 15 were obtained in good to high yields. It should be noted that these reactions proceeded without a loss of the optical purity.

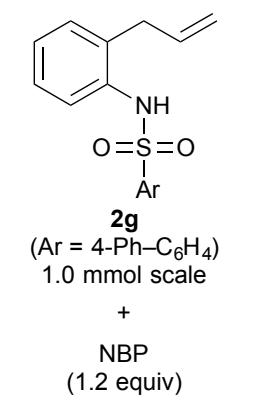

\begin{tabular}{c|c}
$(\mathrm{S})-\mathbf{1 a}$ & $\mathrm{CH}_{2} \mathrm{Cl}_{2}$ \\
$(10 \mathrm{~mol} \%)$ & $-90{ }^{\circ} \mathrm{C}, 24$
\end{tabular}<smiles>O=S(=O)([Ga])N1c2ccccc2C[C@H]1CBr</smiles>

$$
\begin{gathered}
\mathbf{3 g} \\
\left(\mathrm{Ar}=4-\mathrm{Ph}-\mathrm{C}_{6} \mathrm{H}_{4}\right) \\
91 \%, 95: 5 \mathrm{er}
\end{gathered}
$$
$(10 \mathrm{~mol} \%) \downarrow-90{ }^{\circ} \mathrm{C}, 24 \mathrm{~h}$

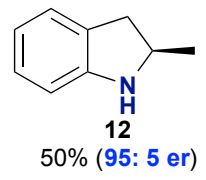

$\mathrm{Ti}(\mathrm{O} i-\mathrm{Pr})_{3}$
$\mathrm{Me}_{3} \mathrm{SiCl}$$\quad \mathrm{THF}$ \begin{tabular}{c|c}
$\mathrm{Me}_{3} \mathrm{SiCl}$ & $50{ }^{\circ} \mathrm{C}, 17 \mathrm{~h}$
\end{tabular}

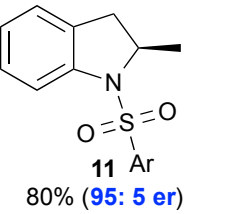

$80 \%$ (95: 5 er)<smiles>NC[C@@H]1Cc2ccccc2N1S(=O)(=O)[AlH2]</smiles>

$90 \%$ (95: 5 er)

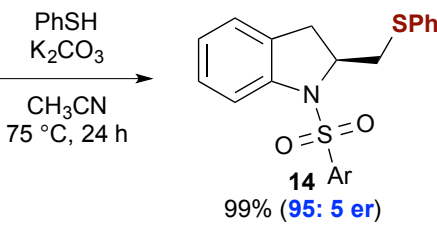

KOAC $\underset{50{ }^{\circ} \mathrm{C}, 7 \mathrm{~h}}{\stackrel{\text { 18-crown-6 }}{\stackrel{\text { DMF }}{\longrightarrow}}}$<smiles>CC(=O)OC[C@@H]1Cc2ccccc2N1S(=O)(=O)[Se][Ba]</smiles>

Scheme 5 Transformations of 2-(bromomethyl)indoline product.

\section{Conclusions}

In summary, we have successfully demonstrated that a chiral bifunctional sulfide catalyst bearing a urea moiety, which was originally developed by our research group, is applicable to enantioselective bromoaminocyclizations. N-(pBiphenylsulfonyl)-2-allylaniline substrates were efficiently transformed to optically active 2-(bromomethyl)indoline derivatives under the influence of our chiral bifunctional 
sulfides. The careful optimization of the $\mathrm{N}$-protecting group on the substrate was key to the achievement of highly enantioselective reactions. Computational studies for the present bromoaminocyclizations have also been investigated to clarify the origin of good stereoselectivities. The additional utility of the present synthetic method was demonstrated in the transformations of the bromoaminocyclization products. Various chiral 2-substituted indolines possessing a wide variety of functional groups were synthesized in a enantioenriched manner. Further applications of our chiral bifunctional sulfides to other catalytic asymmetric transformations are currently underway by our group.

\section{Conflicts of interest}

There are no conflicts to declare.

\section{Acknowledgements}

This work was supported by JSPS KAKENHI Grant Numbers JP19K05480 (for S. S.) and JP17KT0011 (for M. Y.), the Cooperative Research Program of "Network Joint Research Center for Materials and Devices" (20191310 for S. S.), Tokuyama Science Foundation (for S. S.), Takahashi Industrial and Economic Research Foundation (for S. S.), and Shorai Foundation for Science and Technology (for S. S.). This work was the result of using research equipment shared in MEXT Project for promoting public utilization of advanced research infrastructure (Program for supporting introduction of the new sharing system) Grant Number JPMXS0422500320.

\section{Notes and references}

1 For reviews on catalytic asymmetric bromolactonizations and related halofunctionalizations, see: $(a) \mathrm{G}$. Chen and S. Ma, Angew. Chem., Int. Ed., 2010, 49, 8306-8308; (b) C. K. Tan, L. Zhou and Y.-Y. Yeung, Synlett, 2011, 1335-1339; (c) A Castellanos and S. P. Fletcher, Chem. - Eur. J., 2011, 17, 57665776; (d) S. E. Denmark, W. E. Kuester and M. T. Burk, Angew. Chem., Int. Ed., 2012, 51, 10938-10953; (e) U. Hennecke, Chem. - Asian J., 2012, 7, 456-465; (f) C. K. Tan and Y.-Y. Yeung, Chem. Commun., 2013, 49, 7985-7996; $(g)$ K. Murai and H. Fujioka, Heterocycles, 2013, 87, 763-805; (h) C. K. Tan W. Z. Yu and Y.-Y. Yeung, Chirality, 2014, 26, 328-343; (i) S. Zheng, C. M. Schienebeck, W. Zhang, H.-Y. Wang and W. Tang, Asian J. Org. Chem., 2014, 3, 366-376; (j) Y. A. Cheng, W. Z. Yu and Y.-Y. Yeung, Org. Biomol. Chem., 2014, 12, 2333-2343; (k) C. B. Tripathi and S. Mukherjee, Synlett, 2014, 25, 163-169; (I) R. Kristianslund, J. E. Tungen and T. V. Hansen, Org. Biomol. Chem., 2019, 17, 3079-3092.

2 For selected reviews, see: $(a)$ A. Sakakura and K. Ishihara, Chem. Rec., 2015, 15, 728-742; (b) M. H. Gieuw, Z. Ke and Y.Y. Yeung, Chem. Rec., 2017, 17, 287-311; (c) Y. Kawato and Y. Hamashima, Synlett, 2018, 29, 1257-1271; (d) S. E. Denmark, P. Ryabchuk, H. M. Chi and A. Matviitsuk, Org. Synth., 2019, 96, 400-417.

3 (a) R. Nishiyori, A. Tsuchihashi, A. Mochizuki, K. Kaneko, M. Yamanaka and S. Shirakawa, Chem. - Eur. J., 2018, 24, 1674716752; (b) A. Tsuchihashi and S. Shirakawa, Synlett, 2019, 30, 1662-1666; (c) M. Okada, K. Kaneko, M. Yamanaka and S.
Shirakawa, Org. Biomol. Chem., 2019, 17, 3747-3751; (d) R. Nishiyori, J. R. J. Maynard and S. Shirakawa, Asian J. Org. Chem., 2020, 9, 192-196.

4 For reviews on chiral sulfide catalysts, see: $(a)$ V. K. Aggarwal, Synlett, 1998, 329-336; (b) V. K. Aggarwal and C. L. Winn, Acc. Chem. Res., 2004, 37, 611-620; (c) E. M. McGarrigle, E. L. Myers, O. Illa, M. A. Shaw, S. L. Riches and V. K. Aggarwal, Chem. Rev., 2007, 107, 5841-5883; (d) R. Gómez Arrayás and J. C. Carretero, Chem. Commun., 2011, 47, 2207-2211; (e) J. Luo, X. Liu and X. Zhao, Synlett, 2017, 28, 397-401.

5 For recent examples of chiral sulfide catalysts, see: $(a)$ H.-Y. $\mathrm{Wu}$, C.-W. Chang and R.-J. Chein, J. Org. Chem., 2013, 78, 5788-5793; (b) M.-T. Huang, H.-Y. Wu and R.-J. Chein, Chem. Commun., 2014, 50, 1101-1103; (c) Z. Ke, C. K. Tan, F. Chen and Y.-Y. Yeung, J. Am. Chem. Soc., 2014, 136, 5627-5630; (d) Z. Ke, C. K. Tan, Y. Liu, K. G. Z. Lee and Y.-Y. Yeung, Tetrahedron, 2016, 72, 2683-2689; (e) X. Liu, R. An, X. Zhang, J. Luo and X. Zhao, Angew. Chem., Int. Ed., 2016, 55, 5846-5850; (f) Q.-Z. Li, X. Zhang, R. Zeng, Q.-S. Dai, Y. Liu, X.-D. Shen, H.-J. Leng, K.-C. Yang and J.-L. Li, Org. Lett., 2018, 20, 3700-3704; (g) Q. Cao, J. Luo and X. Zhao, Angew. Chem., Int. Ed., 2019, 58, 13151319.

6 (a) M. T. Bovino and S. R. Chemler, Angew. Chem., Int. Ed., 2012, 51, 3923-3927; (b) P. Mizar, A. Burrelli, E. Günther, M. Söftje, U. Farooq and T. Wirth, Chem. - Eur. J., 2014, 20, 13113-13116; (c) S.-N. Yu, Y.-L. Li and J. Deng, Adv. Synth. Catal., 2017, 359, 2499-2508.

7 For selected examples of related catalytic asymmetric aminocyclizations, see: (a) O. Lozano, G. Blessley, T. Martinez del Campo, A. L. Thompson, G. T. Giuffredi, M. Bettati, M. Walker, R. Borman and V. Gouverneur, Angew. Chem., Int. Ed., 2011, 50, 8105-8109; (b) L. Zhou, J. Chen, C. K. Tan and Y.-Y. Yeung, J. Am. Chem. Soc., 2011, 133, 9164-9167; (c) F. Chen, C. K. Tan and Y.-Y. Yeung, J. Am. Chem. Soc., 2013, 135, 12321235; (d) D. Huang, X. Liu, L. Li, Y. Cai, W. Liu and Y. Shi, J. Am. Chem. Soc., 2013, 135, 8101-8104; (e) S. E. Denmark and H. M. Chi, J. Am. Chem. Soc., 2014, 136, 8915-8918; (f) C. B. Tripathi and S. Mukherjee, Org. Lett., 2014, 16, 3368-3371; (g) S. E. Denmark and H. M. Chi, J. Org. Chem., 2017, 82, 38263843; (h) J. Luo, Y. Liu and X. Zhao, Org. Lett., 2017, 19, 34343437.

8 For reviews on asymmetric synthesis of indolines, see: $(a) \mathrm{S}$. Anas and H. B. Kagan, Tetrahedron: Asymmetry, 2009, 20, 2193-2199; (b) D. Liu, G. Zhao and L. Xiang, Eur. J. Org. Chem., 2010, 3975-3984; (c) D. Zhang, H. Song and Y. Qin, Acc. Chem. Res., 2011, 44, 447-457; (d) T. S. Silva, M. T. Rodrigues Jr., H. Santos, L. A. Zeoly, W. P. Almeida, R. C. Barcelos, R. C. Gomes, F. S. Fernandes and F. Coelho, Tetrahedron, 2019, 75, 20632097.

9 (a) J.-D. Chai and M. Head-Gordon, Phys. Chem. Chem. Phys. 2008, 10, 6615-6620; (b) A. V. Marenich, C. J. Cramer and D. G. Truhlar, J. Phys. Chem. B, 2009, 113, 6378-6396.

10 All calculations were performed with the Gaussian 09 package. Computational details are shown in Electronic Supplementary Information (ESI).

11 Although the reason for low yield with moderate enantioselectivity of trifluoromethyl-substituted product $\mathbf{6 c}$ is not clear at this stage, the acidity of $\mathrm{NH}$ on substrates $\mathbf{5}$ would affect the reactivity and enantioselectivity in the present reaction.

12 For examples, see: (a) J. Bermudez, S. Dabbs, K. A. Joiner and F. D. King, J. Med. Chem., 1990, 33, 1929-1932; (b) A. T. Vu, S. T. Cohn, P. Zhang, C. Y. Kim, P. E. Mahaney, J. A. Bray, G. H. Johnston, E. J. Koury, S. A. Cosmi, D. C. Deecher, V. A. Smith, J. E. Harrison, L. Leventhal, G. T. Whiteside, J. D. Kennedy and E. J. Trybulski, J. Med. Chem., 2010, 53, 2051-2062; (c) V. Certal, J.-C. Carry, F. Halley, A. Virone-Oddos, F. Thompson, B. Filoche-Rommé, Y. El-Ahmad, A. Karlsson, V. Charrier, C. 
Delorme, A. Rak, P.-Y. Abecassis, C. Amara, L. Vincent, H. Bonnevaux, J.-P. Nicolas, M. Mathieu, T. Bertrand, J.-P. Marquette, N. Michot, T. Benard, M.-A. Perrin, O. Lemaitre, S. Guerif, S. Perron, S. Monget, F. Gruss-Leleu, G. Doerflinger, H. Guizani, M. Brollo, L. Delbarre, L. Bertin, P. Richepin, V. Loyau, C. Garcia-Echeverria, C. Lengauer and L. Schio, J. Med. Chem., 2014, 57, 903-920. 